\title{
DESERCIÓN EN LA CARRERA DE LICENCIATURA EN EDUCACIÓN Y PEDAGOGÍA EN INGLÉS UNIVERSIDAD DE ATACAMA, CHILE
}

\author{
Marcia Poblete Ríos ${ }^{*}$ \\ Ricardo Vera Martínez
}

\begin{abstract}
RESUMEN:
El presente trabajo da cuenta de aquellas variables asociadas a la deserción estudiantil en la carrera de Licenciatura en Educación y Pedagogía en Inglés, dictada por el Instituto de Idiomas de la Universidad de Atacama.

Se realiza un estudio de las I6 primeras cohortes de la carrera, desde su inicio en I99I, hasta la correspondiente al año 2006. Luego de estudiar detenidamente cada cohorte, pudo determinarse que las variables asociadas más recurrentes de deserción son: cambio de carrera y retiro temporal. En un menor grado de recurrencia se ubican el mal rendimiento, el traslado de ciudad, los problemas económicos y el embarazo. Se ofrecen algunas sugerencias para ser aplicadas como tratamiento remedial, para así promover la disminución de la ocurrencia de deserción en futuras cohortes.
\end{abstract}

Palabras clave: cohortes, deserción, variables asociadas, tratamientos remediales.

\section{DROP-OUTS IN THE EDUCATION LICENTIATE AND ENGLISH TEACHING PROGRAM, UNIVERSITY OF ATACAMA, CHILE}

\begin{abstract}
:
This work surveys the main associated variables of student drop-outs in the program of Education Licentiate and English Teaching offered by the Language Institute of the University of Atacama.

Sixteen cohorts were studied, including the first one in I99I and the corresponding cohort of 2006. It could be determined that the most frequent associated variables of student drop-outs are change of program and temporary leave of absence, whereas poor academic performance, economic problems, change of residence, and pregnancy, have a minor impact on students' decision to quit the program. Some suggestions are given so that student dropouts can be better controlled in future cohorts enrolled in the program.
\end{abstract}

Key words: cohorts, drop-out, associated variables, remedial treatment

* Licenciada en Educación, Profesora de Inglés, Psicopedagoga y Magíster en TEFL. Instituto de Idiomas, Universidad de Atacama, Copiapó, Chile. E-mail: marcia.poblete@uda.cl

* Profesor de Inglés, Magíster en Lingüística. Instituto de Idiomas, Universidad de Atacama, Copiapó, Chile. E-mail: ricardo.vera@uda.cl 
Deserción en la carrera de licenciatura en educación y pedagogía en inglés. Universidad de Atacama, Chile - Marcia Poblete y Ricardo Vera

\section{Introducción}

A raíz del proceso de autoevaluación y posterior acreditación de la carrera de Licenciatura en Educación y Pedagogía en Inglés, se puso de relieve la imperiosa necesidad de contar con registros precisos respecto de las principales variables influyentes que motivaron la deserción de los alumnos de esta carrera.

El presente estudio da cuenta de aquellas variables asociadas a la deserción estudiantil en la carrera de Licenciatura en Educación y Pedagogía en Inglés, dictada por el Instituto de Idiomas de la Universidad de Atacama, Chile.

Considerando el alto número de alumnos que abandonan la carrera por razones desconocidas, se realiza un estudio de las I6 primeras cohortes de la carrera, desde su inicio en I99I, hasta la correspondiente al año 2006. Luego de estudiar detenidamente cada cohorte y de hacer un seguimiento a los estudiantes involucrados, utilizando información proporcionada por medios formales e informales, pudo determinarse que las variables asociadas más recurrentes de deserción son: cambio de carrera y retiro temporal. El mal rendimiento, el traslado de ciudad, los problemas económicos y el embarazo registran un menor grado de recurrencia. Se ofrecen algunas sugerencias para ser aplicadas como tratamiento remedial, para así promover la disminución de la ocurrencia de deserción en futuras cohortes.

\section{Marco Teórico}

\section{I.I Implicancias económicas de la deserción}

El tema de la deserción estudiantil, a nivel universitario, representa un problema que requiere de atención preferencial por sus graves implicancias en el desarrollo de la economía nacional, ya que cada vez que un alumno universitario se convierte en un desertor del sistema, el país pierde una ingente inversión. De allí que los procesos 
de acreditación a los que se están sometiendo las diferentes carreras universitarias acuerden gran relevancia a los esfuerzos realizados para, por una parte, mantener a los estudiantes dentro del sistema y por otra, para introducir medidas de intervención que posibiliten que el tiempo de duración real de los estudios se acerque al tiempo estipulado en el plan de estudios, ya que dicha coincidencia es considerada como un indicador de calidad y eficiencia en la gestión de los programas de estudio.

Desafortunadamente, esta problemática está lejos de tener una solución adecuada en el corto plazo, ya que la mayoría de las instituciones educacionales no han tenido como costumbre de sus administraciones desarrollar algún tipo de seguimiento a los estudiantes desertores para conocer las variables influyentes de abandono y de ese modo, reconocer los síntomas o indicadores que pudiesen evidenciar tempranamente conductas del alumnado tendientes a presentar un desenlace como el ya mencionado. De hecho, al momento de comenzar con la revisión de la literatura para la realización de este trabajo en el año 2005, no fue fácil encontrar investigaciones o reportajes sobre este tema en nuestro país, situación que ha variado en la actualidad debido probablemente a la relevancia intrínseca del tema y su incidencia en el resultado final del proceso de acreditación.

\section{I.2 Principales variables asociadas}

Como se puede apreciar, las variables asociadas a este fenómeno, recurrentes durante los primeros años de la carrera, resultan ser un misterio en este momento debido a que todavía no se ha desarrollado un estudio que confirme la veracidad o falsedad de las presunciones populares respecto de este tema. Sin embargo, frecuentemente se mencionan como factores influyentes en la deserción, el bajo rendimiento académico (reprobación de asignaturas), el embarazo, los problemas socioeconómicos y la vocación de los estudiantes (motivación por continuar en la carrera elegida). Según Muriel Haltern, psiquiatra del Hospital Clínico de la Universidad de Chile, 
Deserción en la carrera de licenciatura en educación y pedagogía en inglés. Universidad de Atacama, Chile - Marcia Poblete y Ricardo Vera

"la deserción en primero o segundo año de carrera es más común, y corresponde a situaciones que tienen que ver con la elección de la carrera, es decir, vocación, madurez del estudiante y otras condiciones secundarias, como oportunidades económicas..." (citado por Juan Pablo Díaz,2004. www.universia.cl/portada/ actualidad/noticia_actualidad.jsp?noticia=58803 ).

Rendimiento académico. Es posible decir que los alumnos que ingresan a las universidades o institutos profesionales chilenos quizás desconocen la rigurosidad de los estudios en la enseñanza superior, no dimensionando adecuadamente el nivel de las exigencias que demandan las asignaturas impartidas en la educación terciaria, que, como es sabido, difieren grandemente de aquellas correspondientes a las asignaturas impartidas en enseñanza media. Según el académico Luis Eduardo González, una de las principales variables asociadas a la deserción está constituida por la falta de preparación con que ingresan los estudiantes a sus estudios universitarios. Esto sugiere la necesidad de mejorar la educación media por un lado y por otro, modificar el proceso de selección de los estudiantes que ingresan a las universidades.

(www.tutoriasarquitectura.cl/art\%EDculos/Art\%EDculo\%20 E1\%20Mercurio\%20-\%20\%201a\%20deserci\%F3n\%20 universitaria.pdf_).

2.2.2. Embarazo. Por otra parte, la variable correspondiente a las alumnas embarazadas hace pensar que dichas alumnas, luego de tener sus hijos, o quizás algunas de ellas antes de terminar sus embarazos, no vuelven al sistema considerando la indiscutida responsabilidad que la maternidad implica, además del gran desgaste físico y económico que esto involucra. Sólo algunas de las alumnas que se encuentran en esta situación realizan el proceso formal de retiro temporal de la universidad, el que figura en el Registro AA de la Facultad de Humanidades y Educación. En la mayoría de los casos, las alumnas solamente descontinúan su asistencia y es el profesor jefe de cohorte quien averigua, también de manera informal, lo sucedido con las alumnas. La revisión de la literatura al respecto no ha permitido reconocer esta variable como determinante de la deserción de los estudios universitarios. 
2.2.3. Problemas económicos. Es sabido que el alumnado que ingresa a la enseñanza superior con puntajes inferiores a 475 puntos vía PSU no tiene acceso a crédito universitario (Memorándum 607, Ley 19.287 Decreto n 5I-0I-2004, Artículo Primero). Esto, sin duda, dificulta el ingreso y permanencia de los alumnos dentro del sistema, especialmente si se considera el hecho de que la mayoría de los alumnos que ingresan a esta universidad proviene de un medio socioeconómico bajo (Informe de Autoevaluación Institucional de la Universidad de Atacama, Fortalezas Gestión Institucional). Un número considerable de los alumnos proviene de otras ciudades e incluso de otras regiones del país, lo que se traduce en un gasto adicional en alojamiento, el que se suma a los gastos considerados fijos, correspondientes a movilización, alimentación y materiales de estudio. Considerando la recurrencia de situaciones como éstas en nuestra universidad, debido principalmente a que gran parte del alumnado posee bajo puntaje de ingreso (Informe de Autoevaluación Carrera de Licenciatura en Educación y Pedagogía en Inglés, p.5I), es que se han creado diferentes sistemas dentro de la institución para ayudar a los alumnos a obtener ingresos que les permitan continuar sus estudios. Existen en este momento dos grandes fuentes de trabajo para el alumnado, la primera consiste en trabajos de verano que ofrece la misma universidad en donde se privilegia a aquellos alumnos que presentan atrasos en los pagos de aranceles o de matrículas. La segunda y muy importante fuente de ingresos consiste en una red de "propineros" que funciona en los diferentes supermercados de la ciudad, iniciativa desarrollada por un alumno de la Facultad de Ingeniería quien está encargado del control y organización de la red. Esta alternativa permite a los alumnos trabajar por turnos y de esta manera generar un ingreso permanente que colabora con sus gastos y disminuye el aporte económico proveniente desde el grupo familiar. Además de estos aportes generados de algún modo dentro de la propia universidad, existen otras alternativas interesantes que colaboran con los alumnos universitarios de escasos recursos, como por ejemplo, la beca Juan Gómez Millas, que permite al solicitante proveniente de la enseñanza municipalizada o subvencionada seguir estudios en la enseñanza superior, permitiendo a un $70 \%$ de los beneficiarios financiar sus estudios. 
Deserción en la carrera de licenciatura en educación y pedagogía en inglés. Universidad de Atacama, Chile - Marcia Poblete y Ricardo Vera

(www.bcn.cl/bibliodigital/pben/informes/estudios_pdf_ informes/nroI28.pdf_).

Por otro lado, el ex Ministro de Educación Sergio Bitar, indicó que a partir del año 2004 el gobierno aplicaría políticas tendientes a mejorar la gestión, la calidad y el financiamiento de la educación superior del país, presentando, entre otras iniciativas, un proyecto cuyo objeto es buscar la aprobación de crédito fiscal para todos los estudiantes, hecho que evidencia la clara intención de proporcionar ayuda económica a los alumnos universitarios.

(www.lidersanantonio.cl/site/edic/20040I I92I000I/ pags/20040I I92330I4.html).

2.2.4. Vocación. Este tema es bastante complejo puesto que se relaciona con la elección que los estudiantes hacen de la carrera que quieren estudiar. No siempre los alumnos egresan de la enseñanza media con una idea clara de sus preferencias; por lo tanto, una adecuada orientación antes del ingreso a la educación superior resulta ser de suma relevancia. Hasta ahora se tenía la percepción de que los estudiantes secundarios nunca tienen toda la información disponible para tomar una decisión bien fundamentada y que en el camino de la educación superior van confirmando su vocación. (http://www.latercera.com/contenido/679_90036_9.shtml)

Si los alumnos ingresan a las instituciones de educación superior desconociendo las asignaturas que conforman la carrera, y con vaga información respecto del campo ocupacional en el que podrán desempeñarse, probablemente experimenten algún grado de frustración o desmotivación con el transcurso del tiempo. Muriel Haltern indica que los alumnos pueden tener expectativas, ideas o deseos de estudiar algo que, al momento de ingresar, notan que no era lo que esperaban, o bien, que no tienen las habilidades o destrezas requeridas para ese estudio. Esto en parte podría ser corregido por las propias instituciones educacionales informando a los interesados tanto de los aspectos atractivos de las carreras, como también de las dificultades de las mismas (citado por Juan 
Pablo Díaz,2004. www.universia.cl/portada/actualidad/noticia_ actualidad.jsp?noticia $=58803$ ).

El Consejo Superior de Educación (CSE) realizó un estudio que indica que un 34\% de los alumnos deserta de las carreras científicas en el primer año, un 32\% en las carreras tecnológicas, un 29\% de las carreras relacionadas con administración y comercio y un 29\% de las carreras vinculadas con arquitectura y arte. En oposición, las que muestran menos deserción son las del rubro salud, educación y ciencias sociales. La alta deserción en las carreras de carácter científico obedecería a la falta de claridad sobre las proyecciones reales de estas carreras y a la necesidad de especializarse $u$ obtener post grados para poder desempeñarse laboralmente y desarrollarse profesionalmente. Según el estudio realizado por el académico Luis Eduardo González, respecto de la repitencia y deserción en la educación superior chilena, los índices en las áreas de salud y educación son del orden del $37 \%$ y 27\%, respectivamente (http://www.cedus.cl/files/REPITENCIA_ DESERCION.pdf).

Aunque el porcentaje de deserción en el área general de la educación no es muy alarmante desde el punto de vista estadístico, la deserción en Pedagogía en Inglés en nuestra universidad se ha convertido en una situación preocupante, pero a tiempo de controlar. Será conveniente entonces, averiguar si existe un aspecto específico de los estudios de una lengua extranjera que incida en el fracaso y deserción estudiantil, motivo que dará pie para una segunda etapa de esta investigación.

Por el momento, nos enfocaremos en realizar un estudio que permita definitivamente manejar información referente a las variables influyentes más recurrentes de deserción universitaria, además del porcentaje real de deserción que afecta a esta carrera en particular. De esta manera, se podrían aplicar medidas de intervención para enfrentar este fenómeno y disminuir en gran medida el problema que aqueja a la totalidad de las instituciones de educación superior. Esto se podría lograr mediante la elaboración y posterior aplicación de un plan remedial especialmente diseñado para este propósito, 
evitando que alrededor de un $40 \%$ de los alumnos que ingresan a educación superior no consiga terminar la carrera que comenzó. (Juan Pablo Díaz, 2004. www.universia.cl/portada/actualidad/ noticia_actualidad.jsp?noticia $=58803$ ).

\section{El Estudio}

Considerando la necesidad de contar con información calificada respecto de las variables asociadas al fenómeno de la deserción en la carrera de Licenciatura en Educación y Pedagogía en Inglés de nuestra universidad, se plantean entonces las siguientes hipótesis:

$\mathrm{H}_{\mathrm{I}}$. Las dificultades específicas en el aprendizaje de un idioma extranjero, el rendimiento académico y problemas vocacionales son las variables asociadas más preponderantes en la deserción de los alumnos de la carrera de Licenciatura en Educación y Pedagogía en Inglés.

$\mathrm{H}_{2}$. La presencia de variables asociadas, tales como la falta de motivación, problemas socioeconómicos y de embarazo suponen un riesgo menor de abandono de los estudios de los alumnos de la carrera de Licenciatura en Educación y Pedagogía en Inglés.

$\mathrm{H}_{3}$. La principal variable asociada a la deserción en alumnos de la carrera de Licenciatura en Educación y Pedagogía en Inglés corresponde a situaciones no contempladas por los investigadores.

\section{Objetivos}

\section{Objetivo General}

Determinar cuáles fueron las principales variables asociadas a la deserción en ex alumnos de la carrera de Licenciatura en Educación y Pedagogía en Inglés de la Universidad de Atacama, con el fin de iniciar la detección precoz de indicadores de probable deserción en los alumnos regulares de la carrera. 
2.I Objetivos Específicos

2.I.I Identificar cuáles fueron las variables asociadas a la deserción en el período contemplado.

2.I.2 Determinar la recurrencia de las variables asociadas a la deserción.

\section{Metodología}

\section{I Tipo y Diseño de Investigación}

Esta es una investigación de carácter descriptivo, no experimental, puesto que estableció como objetivo detectar las problemáticas que actúan sobre la deserción estudiantil (Variable Dependiente) en la carrera de Licenciatura en Educación y Pedagogía en Inglés de la Universidad de Atacama, sin hacer manipulación de la variable dependiente ni de la población objeto de estudio.

\subsection{Variable Dependiente}

Variables asociadas a la deserción estudiantil

Serán consideradas como posibles variables asociadas: la vocación, los problemas económicos, el embarazo y el rendimiento académico deficiente.

\subsection{Muestra}

La muestra estuvo compuesta por ex alumnos que no finalizaron la carrera, quienes fueron consultados mediante una encuesta que apuntó a detectar las problemáticas involucradas en su deserción. Al mismo tiempo, se incluyó la información obtenida desde los profesores jefes de cohorte, quienes funcionan desde el año $200 \mathrm{I}$ en dicho rol. 
Deserción en la carrera de licenciatura en educación y pedagogía en inglés. Universidad de Atacama, Chile - Marcia Poblete y Ricardo Vera

\subsection{Instrumentos para la recolección de datos y procedimiento}

Los instrumentos para la recolección de información fueron elaborados por los investigadores a cargo, quienes se informaron de algunos modelos ya probados que tienen relación con el tipo de investigación en proceso y determinaron el diseño más adecuado para los objetivos de la investigación. Esto derivó en una encuesta que fue aplicada a los ex alumnos de la carrera a quienes se pudo contactar.

Se trabajó con los listados de alumnos matriculados en la carrera de Licenciatura en Educación y Pedagogía en Inglés entre los años I99I (año de inicio de la carrera) hasta el año 2006. Dicha información fue proporcionada por la Unidad de Computación de la Universidad en el primer semestre del 2006 y se logró, en un primer análisis, conocer cuántos alumnos habían egresado de la carrera, cuántos se habían titulado y cuántos no terminaron la carrera.

Se efectuaron llamados telefónicos a todos aquellos ex alumnos a quienes fue posible ubicar, de manera de acceder a sus correos electrónicos. Se enviaron correos a estos alumnos informándolos del proyecto en proceso, solicitando su colaboración con la Universidad, a través del llenado de la encuesta.

Tomando en consideración que la recolección de encuestas no resultó ser lo exitosa que se esperaba, los investigadores decidieron recolectar antecedentes por medio de la revisión de los Registros AA de la Facultad de Humanidades y Educación. Se revisaron los registros existentes desde I99I hasta 2006. Estos registros contienen resoluciones que indican cambios de carrera y postergaciones de estudios, constituyéndose en un aporte importante de antecedentes para la investigación.

Con el fin de complementar la información, se solicitó a los profesores jefes de cohorte aportar antecedentes de aquellos alumnos cuyo ingreso no se encuentra registrado en las nóminas entregadas por el Departamento de Computación de nuestra universidad. De esta manera, se logró información de aquellos alumnos que continuaban 
en el sistema, así como también de aquellos que abandonaron sus estudios.

\section{Análisis de los Datos}

Se observa que de los 262 alumnos ingresados durante este período, I53 de ellos no egresan, es decir, el 58,39\% no termina sus estudios. De este total de 153 alumnos que no terminan sus estudios, se desconocen las razones de abandono de 95, es decir, el 62,09\%. Sólo se conocen las razones de abandono de 58 alumnos, es decir, del 37,91\%.

Es preocupante que se desconozcan las razones de abandono de un porcentaje tan elevado de alumnos. (Cuadro Resumen I. Situación general de ingreso y egreso de alumnos matriculados entre los años I99I y 2000, p.I8).

Se observa que de los 184 alumnos ingresados durante este período, I7I estudiantes aún no egresan, es decir, el 92,93\% no ha terminado sus estudios. De este total de I7 I alumnos que aún no terminan sus estudios, se desconocen las razones de abandono de 39, es decir, el $22,8 \%$. Se conocen las razones de no egreso de I32 alumnos, es decir, del 77,19\%.

Llama la atención que el nivel de desconocimiento de las razones que llevan a los alumnos a no terminar sus estudios aún sea alto, a pesar de que en este periodo se encuentra implementado un sistema de profesores jefes de cohorte, quienes, entre otras de sus funciones, deben mantener un registro de sus alumnos, con el fin de brindarles asesoría respecto de sus decisiones académicas. (Cuadro Resumen 2. Situación general de ingreso y egreso de alumnos matriculados entre los años 2001 y 2006, p.I8).

De un total de 446 alumnos ingresados a la carrera entre los años I99I al 2006, completaron su proceso de formación y titulación un número de 108 jóvenes (24,21\%). Esto significa que hay un número de 324 estudiantes que no terminaron o aún no terminan 
sus estudios, es decir, o bien desertaron del sistema o todavía se encuentran en el programa. Del total de los que abandonaron sus estudios, se desconocen las razones que motivaron el abandono de I $34(4 \mathrm{I}, 35 \%)$ alumnos y se conocen las razones del no egreso de I $90(58,64 \%)$ alumnos.

Es altamente preocupante el alto porcentaje de desconocimiento que existe respecto de las variables asociadas que llevan a los alumnos a abandonar sus estudios. (Cuadro Resumen 3. Situación general de ingreso y egreso de alumnos matriculados entre los años I99I y 2006, p. 2I).

\section{Conclusiones}

Luego de identificar cuáles fueron las principales variables asociadas a deserción en ex alumnos de la carrera de Licenciatura en Educación y Pedagogía en Inglés de la Universidad de Atacama y de determinar su recurrencia en cada caso, lo que fue realizado a través del análisis de los datos obtenidos y de la reflexión respecto de éstos, se puede volver a cada una de las hipótesis planteadas.

Se concluye entonces que " $H_{l}$. Las dificultades específicas en el aprendizaje de un idioma extranjero, el rendimiento académico y problemas vocacionales son las variables asociadas más preponderantes en la deserción de los alumnos de la carrera de Licenciatura en Educación y Pedagogía en Inglés", no se cumple debido a que la variable de mayor recurrencia la constituye el cambio de carrera (34,21\%) seguida del retiro temporal (I I,05\%). Ambas variables corresponden al 45,26\% del total de 190 alumnos de quienes se conoce la razón de su no egreso. La variable "dificultades específicas en el aprendizaje de idiomas", no pudo ser determinada debido a que este aspecto debió conocerse mediante las respuestas a la encuesta aplicada pero debido a la escasa data recogida por esa vía, dicha información no fue considerada representativa.

Respecto de " $H_{2}$. La presencia de ciertas variables asociadas, tales como, la falta de motivación, problemas socioeconómicos y de embarazo suponen un riesgo menor de abandono de los estudios de los alumnos de la carrera de 
Licenciatura en Educación y Pedagogía en Inglés", se pudo comprobar que estas tres variables, sumadas a problemas vocacionales, traslado de ciudad y problemas de rendimiento, constituyen un riesgo menor de deserción, representando sólo el 8,39\% del total de 190 alumnos de los cuales se conoce la razón de su no egreso. Por lo tanto, $\mathrm{H}_{2}$ se cumple parcialmente.

Finalmente, " $H_{3}$. La principal variable asociada a la deserción en alumnos que cursaron la carrera de Licenciatura en Educación y Pedagogía en Inglés corresponde a situaciones no contempladas por los investigadores", se cumple parcialmente, considerando que dentro de las variables con mayor recurrencia se encuentran el cambio de carrera con un 34,21\%, el retiro temporal de alumnos con un I I,05\% y el traslado de ciudad (variable que no resulta de una recurrencia significativa) con un 2,I\% del total de 190 alumnos de los cuales se conoce la razón de su no egreso.

La realización de este estudio permitió conocer que la variable de mayor recurrencia involucrada en la deserción de alumnos de la carrera de Licenciatura en Educación y Pedagogía en Inglés fue provocada por los "cambios de carrera". Si consideramos los antecedentes obtenidos por las diferentes vías de recolección de datos, se puede apreciar que no todos los cambios de carrera han sido debidamente registrados. Se desconoce también la motivación de los alumnos para efectuar dicho cambio y el destino final de esos alumnos.

Hecho similar ocurre con respecto de la variable que la secunda, que corresponde a "retiro temporal" y que también representa un porcentaje importante de deserción. Se hace relevante determinar las razones que los alumnos tienen para efectuar los retiros, con el objeto de determinar si es o no factible que los alumnos vuelvan a concluir sus estudios.

\section{Propuesta de Remediales}

Se presentan, a continuación, algunas propuestas remediales que pretenden contribuir a que en un futuro cercano la cantidad de estudiantes que desertan de la carrera disminuya de modo importante. 
Deserción en la carrera de licenciatura en educación y pedagogía en inglés. Universidad de Atacama, Chile - Marcia Poblete y Ricardo Vera

7.I. Según el resultado de esta investigación, las tutorías de cohorte implicaron un aporte significativo de antecedentes, especialmente los relativos a datos informales. Fue prácticamente ésta la vía para obtener información respecto de aquellos alumnos que se retiraron informalmente de la carrera. Esto deja de manifiesto que el rol del profesor jefe de cohorte debiera reforzarse y consolidarse.

7.2. Otro aspecto importante de considerar es el hechoque existen ingresos de alumnos por vías especiales (becas deportivas, traslado desde otras universidades, etc.), que no aparecen registrados por el Departamento de Computación de la universidad, pues a través de este medio se registran sólo aquellos ingresos vía PSU. El registro oportuno de los ingresos especiales sería de gran ayuda para que los profesores jefes de cohorte conocieran en detalle la totalidad de los alumnos que conforman su cohorte.

7.3. No todos los cambios de carrera realizados por los alumnos se encuentran formalmente tramitados y reconocidos a través de los Registros AA de la Facultad de Humanidades y Educación. Un estudio acucioso de seguimiento, esta vez a aquellos alumnos que han efectuado cambio de carrera, nos permitirá conocer si esos cambios se realizaron dentro de la Facultad de Humanidades y Educación, y de ese modo determinar si las carreras reemplazantes corresponden o no al área de idiomas y/o al área de educación. Del mismo modo, podremos saber si corresponden a otras facultades dentro de nuestra universidad o fuera de ella.

7.4. Deben buscarse estrategias para realizar un seguimiento a aquellos alumnos que efectúan retiro temporal de modo de asegurar un mayor porcentaje de regreso del alumnado a finalizar el proceso de formación.

7.5. Se necesita proponer una segunda etapa de este proyecto, de modo de determinar por un lado, si las razones que motivan a los alumnos al cambio de carrera, dicen relación con dificultades específicas para el aprendizaje de idiomas y por otro, para determinar 
si es posible detectar características conductuales o psicológicas que indiquen probabilidades de deserción en los alumnos.

Cuadro Resumen I. Situación general de ingreso y egreso de alumnos matriculados entre los años I99I y 2000

\begin{tabular}{|c|c|c|c|c|c|c|}
\hline $\begin{array}{c}\text { Año/situación } \\
\text { alumno }\end{array}$ & Ingresan & Egresan & Titulan & $\begin{array}{c}\text { No } \\
\text { egresan }\end{array}$ & $\begin{array}{c}\text { Razón desconocida } \\
\text { no egreso }\end{array}$ & $\begin{array}{c}\text { Razón conocida } \\
\text { no egreso }\end{array}$ \\
\hline I99I & 28 & $\mathrm{I} 9$ & $\mathrm{I} 7$ & 9 & 6 & 3 \\
\hline $\mathrm{I} 992$ & 25 & $\mathrm{I} 5$ & $\mathrm{I} 3$ & $\mathrm{I} 0$ & 3 & 7 \\
\hline $\mathrm{I} 993$ & 32 & $\mathrm{I} 4$ & $\mathrm{I} 4$ & $\mathrm{I} 8$ & 9 & 9 \\
\hline $\mathrm{I} 994$ & $3 \mathrm{I}$ & $\mathrm{I} 3$ & $\mathrm{I} 2$ & $\mathrm{I} 8$ & $\mathrm{I}$ & 8 \\
\hline $\mathrm{I} 995$ & $\mathrm{I} 6$ & 9 & 9 & 7 & $\mathrm{I}$ & 6 \\
\hline $\mathrm{I} 996$ & $\mathrm{I} 0$ & 2 & 2 & 8 & 4 & 4 \\
\hline $\mathrm{I} 997$ & 24 & 7 & 6 & $\mathrm{I} 7$ & 9 & 8 \\
\hline $\mathrm{I} 998$ & 26 & $\mathrm{I} 2$ & 8 & $\mathrm{I} 4$ & $\mathrm{II}$ & 3 \\
\hline $\mathrm{I} 999$ & 36 & $\mathrm{I} 0$ & 7 & 26 & $2 \mathrm{I}$ & 5 \\
\hline 2000 & 34 & 8 & 7 & 26 & $2 \mathrm{I}$ & 5 \\
\hline total & 262 & $\mathrm{I} 09$ & 95 & $\mathrm{I} 53$ & 95 & 58 \\
\hline$\%$ & $\mathrm{I} 00$ & $4 \mathrm{I}, 60$ & 36,25 & $\mathrm{I} 00$ & 62,09 & $37,9 \mathrm{I}$ \\
\hline
\end{tabular}

Cuadro Resumen 2. Situación general de ingreso y egreso de alumnos matriculados entre los años 2001 y 2006.

\begin{tabular}{|c|c|c|c|c|c|c|}
\hline Año/situación & Ingresan & Egresan & Titulan & $\begin{array}{c}\text { No } \\
\text { egresan }\end{array}$ & $\begin{array}{c}\text { Razón desconocida } \\
\text { no egreso }\end{array}$ & $\begin{array}{c}\text { Razón conocida } \\
\text { no egreso }\end{array}$ \\
\hline $200 \mathrm{I}$ & 30 & 7 & 7 & 23 & 8 & 15 \\
\hline 2002 & 35 & 6 & 6 & 29 & 8 & $2 \mathrm{I}$ \\
\hline 2003 & 32 & 0 & 0 & 32 & 6 & 26 \\
\hline 2004 & $3 \mathrm{I}$ & 0 & 0 & $3 \mathrm{I}$ & 7 & 24 \\
\hline 2005 & 26 & 0 & 0 & 26 & 6 & 20 \\
\hline 2006 & 30 & 0 & 0 & 30 & 4 & 26 \\
\hline total & $\mathrm{I} 84$ & $\mathrm{I3}$ & $\mathrm{I3}$ & $\mathrm{I} 7 \mathrm{I}$ & 39 & $\mathrm{I32}$ \\
\hline$\%$ & $\mathrm{I} 00$ & 7,06 & 7,06 & $\mathrm{I} 00$ & 22,8 & 77,19 \\
\hline
\end{tabular}

Cuadro Resumen 3. Situación general de ingreso y egreso de alumnos matriculados entre los años I991 y 2006.

\begin{tabular}{|c|c|c|c|c|c|c|}
\hline $\begin{array}{c}\text { Situación } \\
\text { alumnos } \\
\text { I99I-2006 }\end{array}$ & Ingresan & Egresan & Titulan & $\begin{array}{c}\text { No } \\
\text { egresan }\end{array}$ & $\begin{array}{c}\text { Razón desconocida } \\
\text { no egreso }\end{array}$ & $\begin{array}{c}\text { Razón conocida } \\
\text { no egreso }\end{array}$ \\
\hline Total alumnos & 446 & $\mathrm{I} 22$ & $\mathrm{I} 08$ & 324 & $\mathrm{I} 34$ & $\mathrm{I} 40$ \\
\hline$\%$ & $\mathrm{I} 00$ & 27,35 & $24,2 \mathrm{I}$ & $\mathrm{I} 00$ & $4 \mathrm{I}, 35$ & 58,64 \\
\hline
\end{tabular}


Deserción en la carrera de licenciatura en educación y pedagogía en inglés. Universidad de Atacama, Chile - Marcia Poblete y Ricardo Vera

\section{Bibliografía}

Arrau, F. (2003): Deserción en la educación superior en Chile. [Documento en línea] Disponible en: http://www.bcn.cl/bibliodigital/pben/informes/ estudios_pdf_informes/nroI28.pdf [Consulta 2009, enero 8].

Bitar, S. (2004): Quieren mejorar la educación. [Documento en línea] Disponible en: http://www.lidersanantonio.cl/site/edic/20040I I92I000I/ pags/20040I 192330I4.html [Consulta: 2009 marzo 27].

Díaz, J. (2004): Los por qué de la deserción universitaria. [Documento en línea] Disponible en: http://www.universia.cl/portada/actualidad/noticia_ actualidad.jsp?noticia $=58803$ [Consulta: 2006, octubre I4].

González, L, Uribe, D. y González, S. (2005): Estudio sobre la repitencia y deserción en la educación superior chilena. [Documento en línea] Disponible en: http:/ / www.cedus.cl/files/REPITENCIA_DESERCION.pdf[Consulta: 2009, enero 8].

Informe de Autoevaluación Carrera de Licenciatura en Educación y Pedagogía en Inglés, p. 51

Informe de Autoevaluación Institucional, Fortalezas Gestión Institucional.

Memorándum 607, Ley 19.287 Decreto n 51 01-2004, Artículo Primero.

Miranda, M. (2007): Mineduc trabaja en una fotografía general de la deserción universitaria. [Documento en línea] Disponible en: http://www.tutoriasarquitectura. cl/art\%EDculos/Art\%EDculo\%20E1\%20Mercurio\%20-\%20\%20 1a\%20deserci\%F3n\%20universitaria.pdf [Consulta: 2009, enero 8].

Pavez, K. (2009): Problemas vocacionales son la principal causa de deserción universitaria. [Documento en línea] Disponible en: http://www.latercera.com/ contenido/679_90036_9.shtml [Consulta: 2009, enero 8]. 